\title{
Aplicación de potenciales evocados de estado estable como examen auditivo en una población de jóvenes con diferentes niveles de audición
}

\section{Application of evoked steady-state responses as auditory test on subjects with different auditory degrees}

Pamela Fernández $\mathrm{E}^{1}$, Lisette Marincovich $\mathrm{S}^{1}$, Virginia Olivares $\mathrm{R}^{1}$, Romina Paredes $\mathrm{S}^{1}$, Cristián Godoy B².

\section{RESUMEN}

Introducción: Los potenciales evocados auditivos de estado estable (PEAEE) son respuestas cerebrales frente a estímulos auditivos continuos modulados en amplitud y/ o frecuencia (AM/FM). Este examen puede determinar objetivamente umbrales por frecuencia específica.

Objetivo: Conocer el manejo y describir los resultados de la aplicación de los PEAEE en sujetos con distintos niveles auditivos.

Material y método: Estudio exploratorio y transversal en sujetos de 10 a 30 años con audición normal o pérdida auditiva (55 oídos). El estímulo consistió en un tono puro $(0,5,1,2$ y $4 \mathrm{kHz})$ a $46 \mathrm{~Hz}$ AM/FM.

Resultados: Se obtuvo alta correlación entre la audiometría y PEAEE, tanto en el promedio $(r=0,980)$ como en cada frecuencia evaluada. Los umbrales de PEAEE fueron superiores a los audiométricos y la diferencia promedio fue $23 \mathrm{~dB} H \mathrm{HL}$ en normoyentes y $13 \mathrm{~dB} H \mathrm{HL}$ en hipoacúsicos.

Conclusiones: Los umbrales de PEAEE en sujetos hipoacúsicos fueron más cercanos a los audiométricos que en normoyentes. Dada la alta correlación entre la audiometría y PEAEE, éste podría incorporarse en la evaluación clínica, siendo un complemento en la detección y estudio de patologías auditivas.

Palabras clave: Potencial evocado auditivo de estado estable, frecuencia de modulación, umbral electrofisiológico de PEAEE, umbral audiométrico estimado.

\begin{abstract}
Introduction: Auditory steady-state responses (ASSR) correspond to brain responses evoked by continuous auditory stimuli, modulated in amplitud and/or frequency (AM/FM). This test can objectively determined specific frequency thresholds.

Aim: To understand the procedures and to describe the results of the ASSR test application on subjects with different auditory degrees.

Material and method: Exploratory and transversal study in 10-to-30-years subjects with normal hearing or hearing loss (55 ears). The stimuli were pure tones $(0.5,1,2$ y 4 $\mathrm{kHz})$ at $46-\mathrm{Hz} \mathrm{AM} / \mathrm{FM}$.
\end{abstract}

1 Tesistas de la carrera de Fonoaudiología de la Universidad de Valparaíso.

2 Fonoaudiólogo del Servicio de Otorrinolaringología del Hospital Dr. Sótero de Río. 
Results: A high correlation coefficient was obtained between audiometry and the ASSR as well in the average $(r=0.980)$ as in each evaluated frequency. The ASSR thresholds presented higher values than the audiometrical thresholds. The average difference were $23 \mathrm{~dB} H \mathrm{H}$ in normally hearing subjects and $13 \mathrm{~dB} H \mathrm{H}$ in hearingimpaired subjects.

Conclusion: The ASSR threshold in the hearing-impaired group were closer to the audiometrical thresholds than in the normally hearing group. Due to the high correlation between audiometry and ASSR, it can be considered its incorporation in the clinical evaluation, bestowing a complementary value in the detection of auditory pathologies.

Key words: Auditory steady-state responses, frequency of modulation, electrophysiological ASSR thresholds, estimated audiometrical thresholds.

\section{INTRODUCCIÓN}

En la actualidad, el método más utilizado en la evaluación objetiva de la audición es el registro de las respuestas de latencia corta de tronco cerebral mediante un estímulo clic (PEATC). Este examen es aplicado a pacientes que no pueden responder a la audiometría convencional. Sin embargo, el estímulo del PEATC es de breve duración y abarca un espectro de frecuencias muy amplio y poco específico; entre 2 y $4 \mathrm{kHz}{ }^{1}$. Lo anterior determina la necesidad de investigar nuevas técnicas que permitan evaluar los umbrales auditivos en un mayor rango de frecuencias y de manera más específica.

El potencial evocado auditivo de estado estable (PEAEE) aparece como una alternativa frente a las desventajas que presenta el PEATC. Los PEAEE son respuestas electrofisiológicas evocadas por un estímulo acústico continuo, el cual es modulado por una frecuencia distinta a la de estimulación. Dicha respuesta posee dos componentes; amplitud y fase, los cuales permanecen constantes en el tiem$\mathrm{po}^{2}$. El estímulo continuo es un tono puro denominado frecuencia portadora $(F C)$ y representa a las frecuencias de prueba específica, que van desde 125/ 250 a $8.000 \mathrm{~Hz}^{3}$. Adicionalmente, la frecuencia portadora es modulada a una cierta tasa, la cual se conoce como frecuencia de modulación (Mf) y puede seleccionarse dentro de un rango de 4 a $110 \mathrm{~Hz}^{4}$. Un tono continuo puede ser modulado en amplitud $(A M)$, en frecuencia (FM) 0 en ambas (MM)2. La modulación mixta (MM) genera respuestas de mayor amplitud que la modulación $\mathrm{AM}^{5}$. Las células ciliadas realizan una rectificación del estímulo acústico que cancela los componentes de alta frecuencia. Esto permite que las neuronas alejadas de la cóclea puedan ser más activas a la frecuencia de modulación.

La detección de la respuesta de estado estable se basa en la identificación de una onda desde la actividad eléctrica cerebral de base, mediante la transformada rápida de Fourier (FFT), que convierte los componentes del dominio del tiempo al dominio de la frecuencia. Se observa así, en el espectrograma, un peak que representa la periodicidad de la respuesta de estado estable y que puede ser identificado fácilmente con métodos estadísticos que lo diferencian del ruido basal del EEG. De esta manera, la identificación es automática y objetiva ${ }^{6}$. Uno de los estadígrafos más utilizados es la coherencia de fase (PC), cuyo principio básico es que la latencia de la respuesta estado-estable se corresponde con la Mf. Las respuestas obtenidas por los PEAEE pueden variar según diversos factores. La frecuencia de modulación y edad son parámetros que influyen directamente. Se plantea que con el uso de bajas $M f s(40 \mathrm{~Hz})$ en adultos despiertos y altas Mfs (sobre $70 \mathrm{~Hz}$ ) en bebes sedados o dormidos, se obtienen umbrales más cercanos a los audiométricos ${ }^{7,8}$. Aunque no se ha estudiado en forma acabada, se observa que tanto el ruido ambiental como el tiempo de registro de PEAEE pueden influir en la obtención de respuestas. En la actualidad, no existe un consenso sobre las condiciones óptimas en el procedimiento clínico de los PEAEE9. En resumen, el PEAEE es una técnica doblemente objetiva, tanto en la obtención como en el análisis de la respuesta, que permite obtener umbrales auditivos específicos para cada frecuencia. La aplicación de PEAEE permitiría conocer el grado de pérdida por frecuencia específica en hipoacusias severas y profundas para una temprana rehabilita- 
ción auditiva ${ }^{2,10}$. La principal desventaja de este examen consiste en la gran variabilidad de los resultados en adultos normoyentes, especialmente en la frecuencia $500 \mathrm{~Hz}$.

Debido a la escasez de estudios publicados en Chile que exploren el manejo de los PEAEE y a la potencial ventaja que presenta este examen dentro de la evaluación audiológica, el presente estudio tiene por objetivo conocer el manejo, funcionamiento y respuesta al examen potencial evocado auditivo de estado estable en una población de jóvenes con diferentes niveles de audición.

\section{MATERIAL Y MÉTODO}

Se realizó un estudio de tipo exploratorio, descriptivo y de corte transversal. La selección de la muestra fue de modo no probabilístico, de tipo informal y arbitraria. En una primera etapa se exploraron las condiciones ideales de aplicación del examen PEAEE y el comportamiento del sistema GSI Audera. En base a un estudio piloto con 11 sujetos jóvenes normoyentes, no incluidos en los resultados, se creó una pauta de aplicación de PEAEE que permitió evaluar a los sujetos de la muestra en forma homogénea.

La muestra se conformó de 29 sujetos entre 10 y 30 años (16 hombres y 13 mujeres) con un total de 55 oídos evaluados pertenecientes a internos normoyentes y pacientes con diagnóstico de hipoacusia sensorioneural del Servicio de Otorrinolaringología del Hospital Sótero del Río.

A cada individuo se le presentó un consentimiento informado y luego se aplicó una anamnesis para obtener los antecedentes auditivos. Se realizó también una otoscopía e impedanciometría (equipo Madsen, Zodiac 901) para descartar patologías que comprometan al oído medio. Luego se obtuvo umbrales psicoacústicos aéreos para las frecuencias $0.5,1,2$ y $4 \mathrm{kHz}$, mediante una audiometría (audiómetro Madsen, Midimate 602). En esta investigación se consideró el promedio de las frecuencias evaluadas para determinar si el paciente presentaba audición normal (entre 0 y $20 \mathrm{~dB} \mathrm{HL}$ ) 0 hipoacusia $(>20 \mathrm{~dB} \mathrm{HL})^{11}$.

Como medida objetiva de la audición se utilizaron PEATC y PEAEE (sistema GSI Audera, GrasonStadler). Para la aplicación de tales exámenes se procedió a limpiar la piel para lograr una adecuada impedancia $(<5 \mathrm{~K} \Omega)$. Los electrodos se ubicaron según la siguiente configuración: electrodo positivo $(+)$ en frente alta, electrodos negativos (-) derecho e izquierdo en mastoides ipsilaterales y electrodo común o tierra sobre el puente de la nariz.

En la evaluación mediante PEAEE se utilizó un estímulo modulado en amplitud y frecuencia (AM/ FM) a una tasa de $46 \mathrm{~Hz}$, con $100 \%$ de profundidad en $\mathrm{AM}$ y $10 \%$ en FM. Las frecuencias portadoras fueron $1 \mathrm{kHz}, 4 \mathrm{kHz}, 0,5 \mathrm{kHz}$ y $2 \mathrm{kHz}$ presentadas en este orden en forma monoaural mediante fonos de inserción. Para la búsqueda de umbrales de PEAEE se utilizó el método descendente. Se obtienen así dos valores, uno correspondiente al umbral electrofisiológico (U.E PEAEE) y otro al umbral audiométrico estimado (U.A.E.) dado sólo por el sistema GSI Audera.

El análisis estadístico de los datos fue realizado con el software SPSS 12.0 (Statistical Package for the Social Sciences) utilizando las pruebas no paramétricas Test-T y correlación de Pearson. Se consideró un nivel de significancia estadística de ${ }^{*} p<0,05 y{ }^{* *} p<0,01$.

\section{RESULTADOS}

La evaluación audiológica de los sujetos normoyentes arrojó resultados dentro de parámetros de normalidad. Los resultados en sujetos hipoacúsicos fueron: presencia de antecedentes audiológicos, otoscopía e impedanciometría normales, audiometría y PEATC concordantes con pérdida auditiva sensorioneural.

Se presentan los valores en forma independiente para el grupo de sujetos con audición normal y con pérdida auditiva. En la Tabla 1 se observa el promedio \pm desviación estándar (D.E.) de umbrales audiométricos, electrofisiológicos y estimados en normoyentes ( $\mathrm{n}=38$ oídos). Los valores promedios \pm D.E. del grupo de hipoacúsicos ( $n=17$ oídos) se observan en la Tabla 2.

Se relacionaron los umbrales de la audiometría y los umbrales electrofisiológicos de PEAEE mediante la prueba de Pearson, donde se obtuvo una correlación significativa para normoyentes e hipoacúsicos. Sin embargo, en el grupo de hipoacúsicos el valor 
Tabla 1. Promedio $\pm \mathrm{DE}$ de valores umbrales en sujetos normoyentes

\begin{tabular}{|cccc|}
\hline $\begin{array}{c}\text { Frecuencia } \\
(\mathrm{kHz})\end{array}$ & $\begin{array}{c}\text { U.A. } \\
(\mathrm{dB} \mathrm{HL})\end{array}$ & $\begin{array}{c}\text { U.E. PEAEE } \\
(\mathrm{dB} \mathrm{HL})\end{array}$ & $\begin{array}{c}\text { U.A.E. PEAEE } \\
(\mathrm{dB} \mathrm{HL})\end{array}$ \\
\hline 0,5 & $9,74 \pm 5,81$ & $32,63 \pm 8,44$ & $6,84 \pm 10,10$ \\
1 & $7,76 \pm 5,78$ & $28,82 \pm 6,20$ & $12,37 \pm 7,05$ \\
2 & $4,34 \pm 5,72$ & $26,97 \pm 6,21$ & $11,71 \pm 6,50$ \\
4 & $6,05 \pm 5,09$ & $31,84 \pm 8,42$ & $7,37 \pm 10,12$ \\
Promedio & $6,97 \pm 4,39$ & $30,07 \pm 4,85$ & $9,57 \pm 5,71$ \\
\hline
\end{tabular}

U.A.: umbral audiométrico; U.E. PEAEE: umbral electrofisiológico de PEAEE; U.A.E. PEAEE: umbral audiométrico estimado de PEAEE.

Tabla 2. Promedio \pm DE de valores umbrales en sujetos hipoacúsicos

\begin{tabular}{|cccr|}
\hline $\begin{array}{c}\text { Frecuencia } \\
(\mathrm{kHz})\end{array}$ & $\begin{array}{c}\text { U.A. } \\
(\mathrm{dB} \mathrm{HL})\end{array}$ & $\begin{array}{c}\text { U.E.PEAee } \\
(\mathrm{dB} \mathrm{HL})\end{array}$ & \multicolumn{1}{c|}{$\begin{array}{c}\text { U.A.E PEAee } \\
(\mathrm{dB} \mathrm{HL})\end{array}$} \\
\hline 0,5 & $77,94 \pm 25,13$ & $93,53 \pm 22,76$ & $85,88 \pm 29,01$ \\
1 & $90,88 \pm 28,90$ & $100,88 \pm 25,51$ & $97,06 \pm 30,37$ \\
2 & $92,06 \pm 32,74$ & $106,76 \pm 26,57$ & $103,82 \pm 30,44$ \\
4 & $93,82 \pm 31,15$ & $107,65 \pm 28,62$ & $102,94 \pm 34,91$ \\
Promedio & $88,68 \pm 28,78$ & $102,21 \pm 25,06$ & $97,43 \pm 30,03$ \\
\hline
\end{tabular}

U.A.: umbral audiométrico; U.E. PEAEE: umbral electrofisiológico de PEAEE; U.A.E. PEAEE: umbral audiométrico estimado de PEAEE.

de correlación ( $r=0,908)$ fue mayor que en normoyentes $(r=0,336)$ (Figura $1 \mathrm{~A}$ y B). Al analizar la relación entre los umbrales audiométricos y umbrales audiométricos estimados se observa un fenómeno similar. La correlación entre el umbral audiométrico y umbral audiométrico estimado de PEAEE indica una estrecha relación en el grupo de hipoacúsicos, siendo ésta mucho menor en normoyentes (Figura $1 \mathrm{C}$ y D).

Se evaluó además, la correlación por frecuencia de umbrales audiométricos y electrofisiológicos para el grupo de normoyentes e hipoacúsicos (Tabla 3). Se observa que en normoyentes no hay correlación en ninguna de las frecuencias. Por el contrario, en los sujetos hipoacúsicos, se obtuvo una alta correlación para todas las frecuencias $(p<0,01)$ (Tabla 3$)$. Al analizar las variables umbral audiométrico y umbral audiométrico estimado de PEAEE se observa tam- bién una alta correlación en el grupo de hipoacúsicos en todas las frecuencias evaluadas.

En la Tabla 4 se exponen los umbrales audiométricos y electrofisiológicos de PEAEE en el grupo de normoyentes ( $\mathrm{n}=38$ oídos) y las diferencias entre ambos umbrales. La diferencia entre los umbrales audiométricos y de PEAEE, tanto en el promedio como en cada frecuencia, es estadísticamente significativa $(p<0,01)$.

En el grupo de hipoacúsicos, la diferencia entre el umbral electrofisiológico y el umbral audiométrico no es significativa (Tabla 5).

En la Tabla 6 se reportan los umbrales psicoacústicos y los umbrales estimados dados por el sistema GSI Audera para el grupo de normoyentes. Los valores de umbrales estimados fueron significativamente mayores que los umbrales audiométricos en el promedio $(p<0,05)$. Sin embargo, en las frecuencias 0,5 y $4 \mathrm{kHz}$ no se 


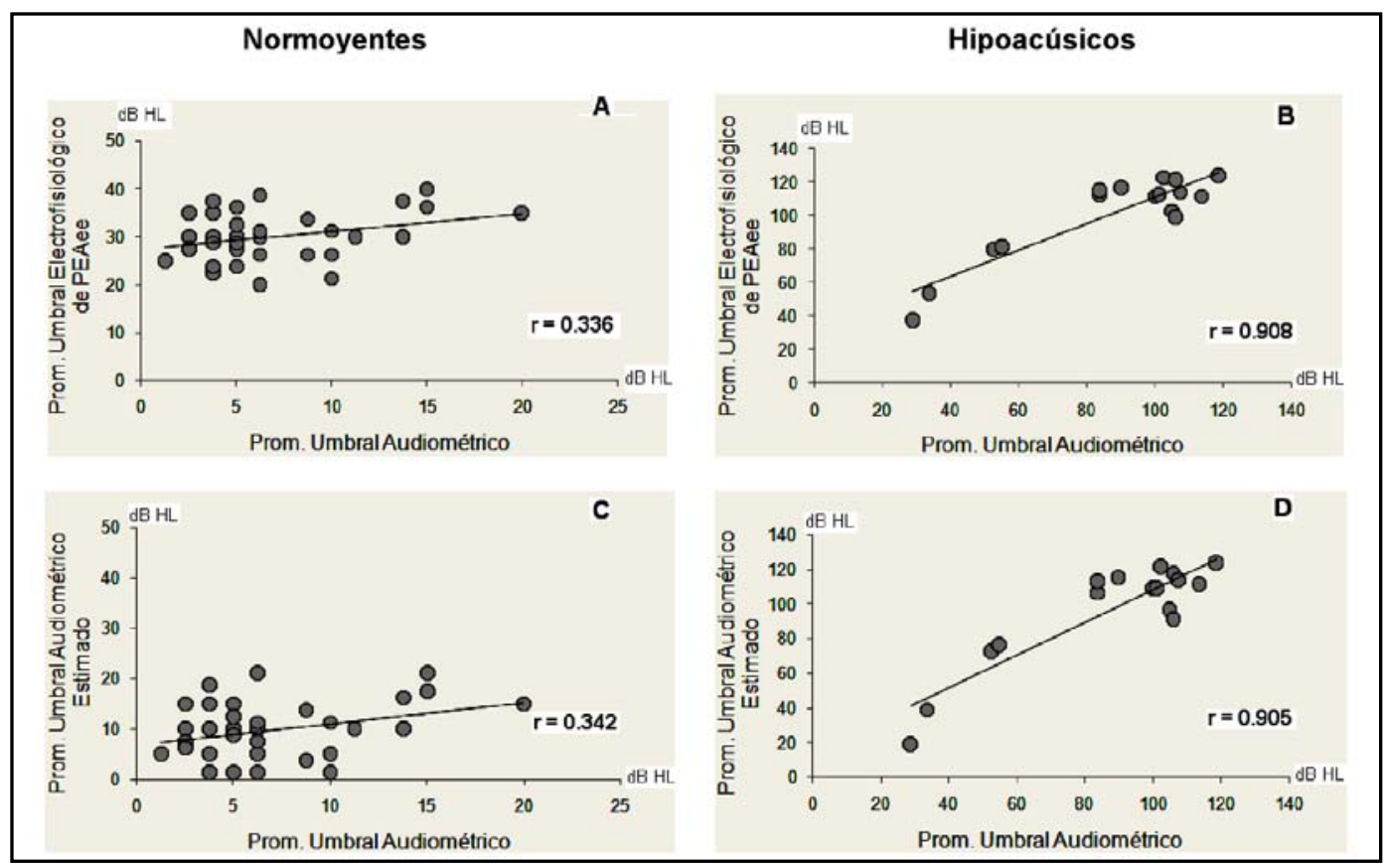

Figura 1. Correlación entre audiometría y PEAEE en sujetos normoyentes e hipoacúsicos. A) Correlación entre umbrales audiométricos y electrofisiológicos de PEAEE en el grupo de normoyentes ( $n=38$ oídos) y $B$ ) en el grupo de hipoacúsicos ( $n=17$ oídos). C) Correlación entre umbrales audiométricos y umbrales estimados de PEAEE en normoyentes y D) hipoacúsicos. r: índice de correlación de Pearson.

encontró una diferencia significativa entre ambos umbrales (Tabla 6).

En el grupo de hipoacúsicos la diferencia promedio entre umbrales estimados y psicoacústicos fue de $8,75 \mathrm{~dB}$ HL. Este valor no representa una diferencia estadísticamente significativa $(p=0,392)$, tendencia que se repite en el análisis por frecuencia (Tabla 7). Además, se observó una alta dispersión de los datos representada por una desviación estándar mayor que el promedio tanto en normoyentes como en hipoacúsicos (Tabla 6 y 7 ).

\section{DISCUSIÓN}

Con el fin de identificar las variables que pudieran influir en el examen de PEAEE se realizó un estudio piloto a partir del cual se propuso un protocolo de

Tabla 3. Correlaciones por frecuencia y por grupo en relación a umbrales audiométricos y umbrales de PEAEE

\begin{tabular}{|ccccc|}
\hline $\begin{array}{c}\text { Frecuencia } \\
(\mathrm{kHz})\end{array}$ & \multicolumn{2}{c}{ r U.A / U.E.PEAEE } & \multicolumn{2}{c|}{ r U.A. / U.A.E.PEAEE } \\
& Normoyentes & Hipoacúsicos & Normoyentes & Hipoacúsicos \\
\hline 0,5 & 0,042 & $0,836^{\star *}$ & 0,000 & $0,796^{\star *}$ \\
1 & 0,226 & $0,891^{\star *}$ & 0,200 & $0,872^{\star *}$ \\
2 & 0,304 & $0,904^{\star *}$ & 0,304 & $0,920^{\star *}$ \\
4 & 0,048 & $0,876^{\star *}$ & 0,148 & $0,878^{\star *}$ \\
\hline
\end{tabular}

U.A.: umbral audiométrico; U.E. PEAEE: umbral electrofisiológico de PEAEE; U.A.E. PEAEE: umbral audiométrico estimado de PEAEE; $r$ índice de correlación de Pearson; ${ }^{* \star}: p<0,01$. 
Tabla 4. Diferencias entre umbral audiométrico y umbral electrofisiológico de PEAEE en normoyentes

\begin{tabular}{|ccccc|}
\hline $\begin{array}{c}\text { Frecuencia } \\
(\mathrm{kHz})\end{array}$ & $\begin{array}{c}\text { U.A. } \\
(\mathrm{dB} \mathrm{HL})\end{array}$ & $\begin{array}{c}\text { U.E. PEAEE } \\
(\mathrm{dB} \mathrm{HL})\end{array}$ & $\begin{array}{c}\mathrm{d}(\mathrm{U} . \mathrm{E} . \mathrm{PEAEE}-\mathrm{U} . \mathrm{A}) \\
\pm \mathrm{DE}(\mathrm{dB} \mathrm{HL})\end{array}$ & $\mathrm{p}$ \\
\hline 0,5 & 9,74 & 32,63 & $22,89 \pm 10,04$ & $0,000^{* *}$ \\
1 & 7,76 & 28,82 & $21,05 \pm 7,46$ & $0,000^{* *}$ \\
2 & 4,34 & 26,97 & $22,63 \pm 7,05$ & $0,000^{* *}$ \\
4 & 6,05 & 31,84 & $25,79 \pm 9,62$ & $0,000^{* *}$ \\
& & 30,07 & $23,09 \pm 5,34$ & $0,000^{* *}$ \\
\hline
\end{tabular}

U.A.: umbral audiométrico; U.E. PEAEE: umbral electrofisiológico de PEAEE; d: diferencia aritmética; p: probabilidad de Test.T; ${ }^{*}: p<0,01 ; n=38$ oídos.

Tabla 5. Diferencias entre umbral audiométrico y umbral electrofisiológico de PEAEE en hipoacúsicos

\begin{tabular}{|ccccc|}
\hline $\begin{array}{c}\text { Frecuencia } \\
(\mathrm{kHz})\end{array}$ & $\begin{array}{c}\mathrm{U} . \mathrm{A} \\
(\mathrm{dB} \mathrm{HL})\end{array}$ & $\begin{array}{c}\text { U.E. PEAEE } \\
(\mathrm{dB} \mathrm{HL})\end{array}$ & $\begin{array}{c}\mathrm{d}(\mathrm{U} . \mathrm{E} . \text { PEAEE }-\mathrm{U} . \mathrm{A}) \\
\pm \mathrm{DE}(\mathrm{dB} \mathrm{HL})\end{array}$ & $\mathrm{p}$ \\
\hline 0,5 & 77,94 & 93,53 & $15,59 \pm 13,91$ & 0,067 \\
1 & 90,88 & 100,88 & $10,00 \pm 13,11$ & 0,292 \\
2 & 92,06 & 106,76 & $14,71 \pm 14,30$ & 0,160 \\
4 & 93,82 & 107,65 & $13,82 \pm 15,06$ & 0,187 \\
Promedio & 88,68 & 102,21 & $13,53 \pm 12,09$ & 0,153 \\
\hline
\end{tabular}

U.A.: umbral audiométrico; U.E. PEAEE: umbral electrofisiológico de PEAEE; d: diferencia aritmética; p: probabilidad de Test.T; $\mathrm{n}=17$ oídos.

Tabla 6. Diferencias entre umbral audiométrico estimado de PEAEE y umbral audiométrico en normoyentes

\begin{tabular}{|ccccc|}
\hline $\begin{array}{c}\text { Frecuencia } \\
(\mathrm{kHz})\end{array}$ & $\begin{array}{c}\text { U.A. } \\
(\mathrm{dB} \mathrm{HL})\end{array}$ & $\begin{array}{c}\text { U.A.E PEAEE } \\
(\mathrm{dB} \mathrm{HL})\end{array}$ & $\begin{array}{c}\text { d (U.A.E.PEAEE }- \text { U.A }) \\
\pm \mathrm{DE}(\mathrm{dB} \mathrm{HL})\end{array}$ & $\mathrm{p}$ \\
\hline 0,5 & 9,74 & 6,84 & $-2,89 \pm 11,37$ & 0,129 \\
1 & 7,76 & 12,37 & $4,61 \pm 8,17$ & $0,002^{* *}$ \\
2 & 4,34 & 11,71 & $7,37 \pm 7,24$ & $0,000^{* *}$ \\
4 & 6,05 & 7,37 & $1,32 \pm 10,95$ & 0,476 \\
Promedio & 6,97 & 9,57 & $2,60 \pm 5,98$ & $0,029^{*}$ \\
\hline
\end{tabular}

U.A.: umbral audiométrico; U.E. PEAEE: umbral audiométrico estimado de PEAEE; d: diferencia aritmética; p: probabilidad de Test.T; ${ }^{*}: p<0,05 ;{ }^{* *}: p<0,01 ; n=38$ oídos.

aplicación de PEAEE. En esta primera aproximación se observó que el ruido ambiental, ruido de artefactos eléctricos, tensión muscular y estado de conciencia del sujeto, producirían una actividad electroencefalográfica aumentada, dificultando la identificación de las respuestas de estado estable. El ruido ambiental actuaría como enmascarante sobre el estímulo, disminuyendo la amplitud de la 
Tabla 7. Diferencias entre umbral audiométrico estimado de PEAEE y umbral audiométrico en hipoacúsicos

\begin{tabular}{|ccccc|}
\hline $\begin{array}{c}\text { Frecuencia } \\
(\mathrm{kHz})\end{array}$ & $\begin{array}{c}\text { U.A. } \\
(\mathrm{dB} \mathrm{HL})\end{array}$ & $\begin{array}{c}\text { U.A.E. PEAEE } \\
(\mathrm{dB} \mathrm{HL})\end{array}$ & $\begin{array}{c}\mathrm{d}(\mathrm{U} . \mathrm{A} . \mathrm{E} . \text { PEAEE }- \text { U.A }) \\
\pm \mathrm{DE}(\mathrm{dB} \mathrm{HL})\end{array}$ & $\mathrm{p}$ \\
\hline 0,5 & 77,94 & 85,88 & $7,94 \pm 17,68$ & 0,399 \\
1 & 90,88 & 97,06 & $6,18 \pm 15,06$ & 0,547 \\
2 & 92,06 & 103,82 & $11,76 \pm 12,86$ & 0,286 \\
4 & 93,82 & 102,94 & $9,12 \pm 16,70$ & 0,427 \\
Promedio & 88,68 & 97,43 & $8,75 \pm 12,85$ & 0,392 \\
\hline
\end{tabular}

U.A.: umbral audiométrico; U.E. PEAEE: umbral audiométrico estimado de PEAEE; d: diferencia aritmética; p: probabilidad de Test.T; $\mathrm{n}=17$ oídos.

respuesta y dificultando su registro desde el EEG ${ }^{12}$. Se observó también, respuestas más estables al utilizar una tasa de modulación de $46 \mathrm{~Hz}$, mientras el paciente se mantuviera despierto. Estos hallazgos corroboran lo planteado por diversos investigadores $^{13-17}$, donde un estímulo de PEAEE modulado a baja frecuencia activaría generadores neurales sensibles al estado de vigilia. La identificación de estas variables permitió determinar las capacidades o limitaciones propias del examen y los parámetros a seguir al momento de evaluar mediante un protocolo de aplicación.

En sujetos con audición normal se obtuvo umbrales de PEAEE similares a estudios previos, tanto en el promedio como en cada frecuencia evaluada $^{12,18,19}$. En presencia de pérdida auditiva se observó una tendencia a umbrales mayores en frecuencias más agudas. Este hecho concuerda con el tipo de curva audiométrica descendente, propia de las hipoacusias sensorioneurales.

Para determinar el grado de adaptabilidad del examen de PEAEE se realizó un análisis de correlación entre los umbrales psicoacústicos entregados por la audiometría y los umbrales de PEAEE. Esto debido a que la audiometría, además de ser un método ampliamente utilizado, entrega umbrales auditivos por frecuencia específica al igual que el PEAEE.

Al comparar los umbrales audiométricos con los umbrales electrofisiológicos de PEAEE, nuestros resultados indican que los pacientes con pérdida auditiva presentan mayor correlación que los normoyentes. Otras investigaciones plantean res- puestas similares, sugiriendo que el PEAEE es me-

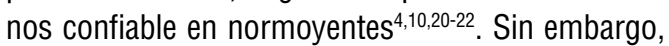
debe considerarse la variable ruido ambiental, la cual dificultaría la percepción del estímulo en individuos normoyentes o con pérdidas auditivas leves. Por otra parte, los umbrales de pacientes hipoacúsicos mostraron una alta correlación en todas las frecuencias evaluadas, siendo éstas semejantes entre sí. Según antecedentes previos ${ }^{10,16,23,24} y$ los aportados en este estudio no existirían variaciones por frecuencia en los PEAEE. En contraste, se han reportado investigaciones donde la frecuencia $0,5 \mathrm{kHz}$ posee menor correlación que otras frecuencias estudiadas. Esta situación se explicaría por el modelo de activación más amplio en la membrana basilar, así como por el tiempo de viaje aumentado hacia la región apical de la cóclea, donde se detectan los sonidos graves. Además el ruido ambiental puede producir un efecto de enmascaramiento en las frecuencias más bajas ${ }^{4,25-30}$. La frecuencia de modulación $(M f)$ también podría ser un factor que influye en la respuesta de PEAEE. Se ha observado que estudios con bajas correlaciones para $0,5 \mathrm{kHz}$, utilizaron estímulos modulados a alta frecuencia. Por el contrario, se han reportado correlaciones similares entre frecuencias al estimular con baja $M f$. En forma particular, se plantea que una $M f$ de $40 \mathrm{~Hz}$ aumentaría la agudeza en la obtención de umbrales en frecuencias bajas, por ejemplo, en $0,5 \mathrm{kHz}^{31}$. Dadas las diferencias metodológicas entre estos estudios, se deben desarrollar investigaciones enfocadas en la Mfy su influencia sobre la respuesta de estado estable. 
Se analizó, también, la relación entre umbrales audiométricos y umbrales estimados de PEAEE que entrega el sistema GSI Audera, donde se mantiene la tendencia de una baja correlación para el grupo de normoyentes y una alta correlación en hipoacúsicos.

En nuestros resultados se aprecia que los umbrales electrofisiológicos de PEAEE son mayores que los umbrales audiométricos. Al igual que en las respuestas de PEATC, existiría una dificultad para detectar potenciales electrofisiológicos de baja amplitud desde el EEG en presencia de ruido ${ }^{32}$. Al analizar las diferencias entre los umbrales electrofisiológicos de PEAEE y los audiométricos en el grupo de normoyentes, se observó una diferencia significativa entre ambos valores. Por el contrario, no se obtuvo diferencias en el grupo de hipoacúsicos, tanto en el promedio como en cada una de las frecuencias evaluadas. Esta similitud entre ambos umbrales concuerda con los altos índices de correlación descritos anteriormente para el mismo grupo y se corresponde con investigaciones previas ${ }^{6,23,31}$. En el grupo de normoyentes se observaron diferencias mayores que las planteadas por otros autores ${ }^{20,24,29,33}$. Esta discrepancia se puede argumentar por la limitación en el control del ruido ambiental al no realizar el examen de PEAEE en una cámara sonoamortiguada, variable que afectaría en mayor grado a sujetos con mejor nivel auditivo.

Los umbrales estimados que entrega el PEAEE corresponden a un valor promedio, dado por una ecuación de regresión en base al umbral electrofisiológico del mismo examen. Esta estimación del umbral audiométrico será más precisa mientras mayor sea la pérdida auditiva ${ }^{23}$. Nuestros hallazgos indican que los valores de umbrales audiométricos estimados son cercanos a los umbrales audiométricos reales en presencia de hipoacusia. Si bien, la desviación estándar en hipoacúsicos $(12,85)$ fue mayor que en el grupo de normoyentes $(5,71)$, se debe considerar que los sujetos presentaban pérdidas auditivas de diversos grados. Por esto se requieren futuros estudios que consideren los grados de pérdida auditiva en relación a la estimación de umbrales audiométricos mediante PEAEE. Es importante señalar que son escazas las investiga- ciones que analizan en profundidad los valores del audiograma estimado del sistema Audera, ya que Ios equipos más utilizados para el registro de PEAEE no ofrecen esta alternativa.

\section{CONCLUSIONES}

En relación a los parámetros y condiciones de aplicación del examen de PEAEE, es necesario considerar las características propias del equipo y del sujeto a evaluar. En individuos adultos con un estado de alerta vigil, se obtienen respuestas más estables con una $M f$ de $46 \mathrm{~Hz}$ al evaluar las frecuencias 0,5, 1, 2 y $4 \mathrm{kHz}$.

Umbrales electrofisiológicos de PEAEE y audiométricos presentan una alta correlación en todas las frecuencias evaluadas. Los valores de umbrales electrofisiológicos son mayores que los umbrales audiométricos y esta diferencia disminuye en presencia de pérdida auditiva. Los resultados obtenidos en este estudio corroboran lo descrito por otras investigaciones.

Los umbrales audiométricos estimados son más cercanos a los umbrales psicoacústicos en presencia de hipoacusia, de esta forma el audiograma estimado entregado por el sistema GSI Audera podría utilizarse como una guía en la estimación del nivel de pérdida auditiva. Sin embargo, se requieren estudios en la población chilena para determinar algoritmos específicos por frecuencia que extrapolen los valores en forma más certera.

Este estudio refleja que la técnica de PEAEE, como instrumento clínico de evaluación, ofrece un valor complementario importante dentro del conjunto de pruebas audiológicas, ya que puede acercarse objetivamente a la configuración, grado y tipo de hipoacusia.

Como examen objetivo, la mayor aplicación clínica del examen de PEAEE apunta a pacientes en los que no es posible determinar el nivel auditivo mediante pruebas subjetivas. En el caso de neonatos con deficiencia auditiva, la detección e intervención temprana es esencial para el desarrollo del lenguaje y el habla. 


\section{BIBLIOGRAFÍA}

1. Lehnhardt E. Práctica de la Audiometría. Madrid. Editorial Médica Panamericana S. A. 1992; 6 Edición.

2. Martínez A, Alañón ma, Ayala l, Álvarez ab, Miranda MT, SaInz M. Comparative study between auditory steady-state responses, auditory brain-stem responses, and liminar tonal audiometry. Acta Otorrinolaringol Esp 2007; 58: 290-5.

3. Ménard M, Gallego S, Berger-Vachon C, Collet L, Thal-Van H. Relationship between loudness growth function and auditory steady-state response in normal-hearing subjects. Hear Res 2008; 235: 105-13.

4. Martínez-Beneito P, Morant A, Pitarch Mi, García FJ, Marco J. Potenciales evocados auditivos de estado estable a multifrecuencia como técnica de determinación de umbrales auditivos. Acta Otorrinolaringol Esp 2002; 53: 707-17.

5. John MS, Purcell DW, DimitriJevic A, Picton TW. Advantages and caveats when recording steady-state responses to multiple simultaneous stimuli. J Am Acad Audiol 2002; 13: 246-59.

6. Canale A, lacilla M, Cavalot al, Albera R. Auditory steady-state responses and clinical applications. Eur Arch Otorhinolaryngol 2006; 263: 499-503.

7. Tomlin D, Rance G, Graydon K, Tsialios I. A comparison of $40 \mathrm{~Hz}$ auditory steady-state response (ASSR) and cortical auditory evoked potential (CAEP) thresholds in awake adult subjects. Int J Audiol 2006; 45: 580-8.

8. Cabello P, Caro J. Audiometría de Estado Estable. Steady state audiometry. Rev otorrinolaringol Cir Cab-Cuello 2007; 67: 162-6.

9. Luts $H$, Wouters J. Hearing assessment by recording multiple auditory steady-state responses: the influence of test duration. Int $J$ Audiol 2004; 43: 471-8.

10. Ahn JH, Lee HS, Kim YJ, Yoon TH, Chung JW. Comparing pure-tone audiometry and auditory steady state response for the measurement of hearing loss. Otolaryngol Head Neck Surg 2007; 136: 966-71.

11. Garantías Explícitas en Salud. Guía Clínica MINSAL. (2005). Hipoacusia Neurosensorial
Bilateral del Prematuro. Disponible en http:// www.supersalud.cl/documentacion/569/ articles-635_guia_clinica.pdf

12. Kel J, Smith D, Joseph S, Stopa J, Kang S, Darnell. R. Effects of ambient acoustic noise on the auditory steady-state response thresholds in normally hearing adults. Audiol Neurootol 2008; 13: 13-8.

13. Cohen LT, Rickards FW, Clark GM. A comparison of steady-state evoked-potentials to modulated tones in awake and sleeping humans. J Acoust Soc Am 1991; 90: 2467-79.

14. Petitot C, Collet L, Durrant JD. Auditory steady-state responses (ASSR): effects of modulation and carrier frequencies. Int $J$ Audiol 2005; 44: 567-73.

15. Leigh-Paffenroth ED, Fowler CG. Amplitudemodulated auditory steady-state responses in younger and older listeners. J Am Acad Audiol 2006; 17: 582-97.

16. Van der ReiJden CS, Mens lhM, Snik AFM. Frequency-specific objective audiometry: Toneevoked brainstem responses and steady-state responses to $40 \mathrm{~Hz}$ and $90 \mathrm{~Hz}$ amplitude modulated stimuli. Int J Audiol 2006; 45: 40-5.

17. Griskova I, Morup M, Parnas J, Ruksenas 0, ARNFRED SM. The amplitude and phase precision of $40 \mathrm{~Hz}$ auditory steady-state response depend on the level of arousal. Exp Brain Res 2007; 183: 133-8.

18. Pérez-Ábalo mC, Torres A, Savio G, Suárez Ee. Los potenciales evocados auditivos de estado estable a múltiples frecuencias y su valor en la evaluación objetiva de la audición. Auditio: Revista Electrónica de Audiología 2003; 2: 42-50.

19. Swanepoel D, Hugo R. Estimations of auditory sensitivity for young cochlear implant candidates using the ASSR: preliminary results. Int J Audiol 2004; 43: 377-82.

20. Herdman AT, Stapells DR. Thresholds determined using the monotic and dichotic multiple auditory steady-state response technique in normal-hearing subjects. Scand Audiol 2001; 30: 41-9.

21. Rance G, Briggs RJS. Assessment of hearing in infants with moderate to profound impairment: The Melbourne experience with auditory steady-state evoked potential testing. Ann Otol Rhinol Laryngol 2002; 111: 22-8. 
22. Rance G, Rickards F. Prediction of hearing threshold in infants using auditory steady-state evoked potentials. J Am Acad Audiol 2002; 13: 236-45.

23. Rance G, Rickards FW, Cohen LT, De Vidi S, Clark GM. The automated prediction of hearing thresholds in sleeping subjects using auditory steady-state evoked potentials. Ear Hear 1995; 16: 499-507.

24. Van Maanen A, Stapells DR. Comparison of multiple auditory steady-state responses (80 versus $40 \mathrm{~Hz}$ ) and slow cortical potentials for threshold estimation in hearing-impaired adults. Int J Audiol 2005; 44: 613-24.

25. LiNS OG, PICTON TW, Boucher BL et al. Frequency-specific audiometry using steadystate responses. Ear Hear 1996; 17: 81-96.

26. Pérez-Ábalo MC, Savio G, Torres A, Martín V, Rodríguez E. Steady state responses to multiple amplitude-modulated tones: An optimized method to test frequency-specific thresholds in hearing-impaired children and normal-hearing subjects. Ear Hear 2001; 22: 200-11.

27. Picton TW, Durieux-Smith A, Champagne SC et al. Objective evaluation of aided thresholds using auditory steady-state responses. J Am Acad Audiol 1998; 9: 315-31.
28. Dimitrijevic A, John S, Van Roon P, Purcell DW, Adamonis J. Estimating the audiogram using multiple auditory steadystate responses. $\mathrm{J} \mathrm{Am}$ Acad Audiol 2002; 13: 205-24.

29. Attias J, Buller N, Rubel Y, Raveh E. Multiple auditory steady-state responses in children and adults with normal hearing, sensorineural hearing loss, or auditory neuropathy. Ann Otol Rhinol Laryngol 2006; 115: 268-76.

30. D'Haenens W, Dhooge I, De Vel E, Maes L, BOCKStael A, Vinck BM. Auditory steady-state responses to $M M$ and exponential envelope AM2/FM stimuli in normal-hearing adults. Int $\mathrm{J}$ Audiol 2007; 46: 399-406.

31. Tlumak Al, Rubinstein E, Durrant JD. Metaanalysis of variables that affect accuracy of threshold estimation via measurement of the auditory steady-state response (ASSR). Int $\mathrm{J}$ Audiol 2007; 46: 692-710.

32. Barajas JJ, Zenker F. Potenciales Evocados Auditivos Continuos. Auditio: Revista Electrónica de Audiología 2002; 1: 20-4.

33. Kaf WA, Sabo DL, Durrant JD, Rubinstein E. Reliability of electric response audiometry using $80 \mathrm{~Hz}$ auditory steady-state responses. Int J Audiol 2006; 45: 477-86. 